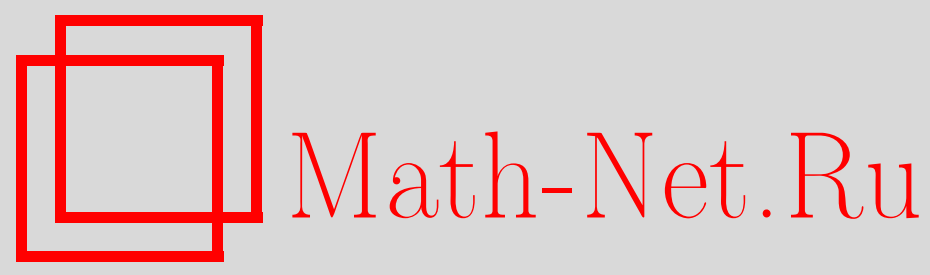

В. А. Краснов, О поверхности Фано вещественной трехмерной $M$-кубики, Матем. заметки, 2005, том 78, выпуск $5,710-717$

DOI: https://doi.org/10.4213/mzm2634

Использование Общероссийского математического портала Math-Net.Ru подразумевает, что вы прочитали и согласны с пользовательским соглашением http://www.mathnet.ru/rus/agreement

Параметры загрузки:

IP: 54.224 .187 .69

26 апреля 2023 г., $12: 42: 43$

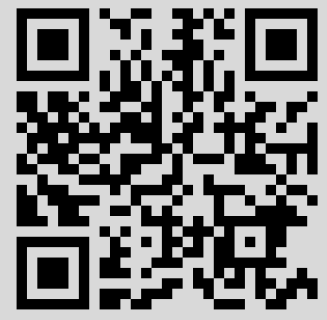




\title{
О ПОВЕРХНОСТИ ФАНО ВЕЩЕСТВЕННОЙ ТРЕХМЕРНОЙ $M$-КУБИКИ
}

\section{В. А. Краснов}

\begin{abstract}
Доказьвается, что поверхность Фано, параметризующая множество прямых на вещественном трехмерном $M$-многообразии третьей степени, сама является $M$-поверхностью. Также справедливо обратное утверждение. При доказательстве используются результаты из работы $Г$. Клеменса и $\Phi$. Гриффитса о промежуточном якобиане трехмерной комплексной кубики.

Библиографияя: 8 названий.
\end{abstract}

Введение. Далее $X$ - комплексное алгебраическое многообразие в комплексном проективном пространстве $\mathbb{P}^{4}$, заданное уравнением

$$
P\left(x_{0}, x_{1}, x_{2}, x_{3}, x_{4}\right)=0
$$

где $P$ - однородньй многочлен третьей степени с вещественными коэффициентами. Мы будем предполагать, что многообразие $X$ неособое. Через $\mathbb{R} X$ будем обозначать множество вещественных решений уравнения (1); оно всегда непустое и состоит из одной или двух компонент связности. Через $F_{X}=F$ будем обозначать поверхность Фано кубики $X$, которая параметризует множество прямьх на $X$. Она будет также неособым многообразием, определенным над полем вещественных точек, так как многочлен $P$ с вещественными коэффициентами. Поэтому определено множество вещественных точек $\mathbb{R} F$ комплексной поверхности $\Phi$ ано $F$, параметризующее множество прямых на $\mathbb{R} X$. Это множество также всегда непустое, так как на вешественной кубике всегда имеются вещественные прямые. На многообразиях $X, F$ определена инволюция комплексного сопряжения $\tau$, причем множества вещественных точек $\mathbb{R} X, \mathbb{R} F$ совпадают со множествами неподвижных точек этой инволюции: $\mathbb{R} X=X^{\tau}, \mathbb{R} F=F^{\tau}$. Поэтому имеют место неравенства Гарнака-Тома

$$
\begin{aligned}
\operatorname{dim}_{\mathbb{F}_{2}} H^{*}\left(\mathbb{R} X, \mathbb{F}_{2}\right) & \leqslant \operatorname{dim}_{\mathbb{F}_{2}} H^{*}\left(X, \mathbb{F}_{2}\right), \\
\operatorname{dim}_{\mathbb{F}_{2}} H^{*}\left(\mathbb{R} F, \mathbb{F}_{2}\right) & \leqslant \operatorname{dim}_{\mathbb{F}_{2}} H^{*}\left(F, \mathbb{F}_{2}\right),
\end{aligned}
$$

где $H^{*}\left(-, \mathbb{F}_{2}\right)=\bigoplus_{q} H^{q}\left(-, \mathbb{F}_{2}\right)$ и $\mathbb{F}_{2}=\{0,1\}$ - поле из двух элементов. Напомним, что каждое из многообразий $(X, \tau),(F, \tau)$ назьвается $M$-многообразием, если соответствуюшее ему неравенство (2) или (3) становится равенством. Главная задача данной заметки - показать, что справедлива следующая

Работа выполнена при финансовой поддержке фонда INTAS-OPEN, грант № 2000-269. 
Теорема 0.1. Поверхность Фано $(F, \tau)$ будет тогда и только тогда $M$-поверхностью, когда кубика $(X, \tau)$ является $M$-многообразием.

Заметим, что аналогичное утверждение справедливо для кубической поверхности. Как известно, на ней имеется 27 прямых, причем они будут все вещественными тогда и только тогда, когда кубическая поверхность является $M$-поверхностью. Вполне возможно, что аналогичная теорема справедлива и для кубики размерности больше трех. Решение этой задачи для трехмерной кубики упрощается, так как в работе [1] имеются почти все необходимые результаты по топологии и геометрии поверхности Фано $F$. Прежде чем доказьвать теорему 0.1, мы приведем без доказательства некоторые результаты из работы [1], а также дополним их уточняющими утверждениями, которые потребуются при доказательстве теоремы 0.1. Этим целям посвящен первый пункт данной статьи. В следующем пункте проводится непосредственное доказательство теоремы 0.1. Это делается с использованием эквивариантных когомологий.

1. Предварительные факты о комплексной поверхности Фано. В этом пункте мы опишем структуру кольца когомологий $H^{*}(F, \mathbb{Z})$. В работе [1] это кольцо почти полностью вычислено. Это делается с помощью метода Лефшеца. А именно, данная кубика $X$ включается в линейное семейство кубик $X_{t}, t \in \mathbb{P}^{1}$, которое представляет собой пучок гиперплоских сечений четырехмерной кубики, причем особые сечения содержат только одну простую двойную точку, а других особых точек нет. Из семейства кубик $X_{t}$ получается семейство поверхностей $\Phi$ ано $F_{t}$. В работе [1] сначала изучается поверхность $\Phi$ ано $F_{0}$ особой кубики $X_{0}$, а затем поверхность $\Phi$ ано $F_{t}$ неособой кубики $X_{t}$. Это делается с помошью деформации $F_{t}$ в $F_{0}$.

Итак, через $X_{0}$ мы будем обозначать трехмерную комплексную кубику в $\mathbb{P}^{4}$ с одной простой двойной точкой, а других особых точек на ней нет. Будем предполагать, что особая точка $p_{0}$ имеет однородные координаты $(1: 0: \cdots: 0)$. В аффинных координатах

$$
y_{1}=\frac{x_{1}}{x_{0}}, \quad y_{2}=\frac{x_{2}}{x_{0}}, \quad y_{3}=\frac{x_{3}}{x_{0}}, \quad y_{4}=\frac{x_{4}}{x_{0}}
$$

уравнение многообразия $X_{0}$ примет вид

$$
\sum_{1 \leqslant i, j \leqslant 4} a_{i j} y_{i} y_{j}+\sum_{1 \leqslant i, j, k \leqslant 4} b_{i j k} y_{i} y_{j} y_{k}=0
$$

где матрища $\left(a_{i j}\right)$ невырожденная, так как мы предполагали, что особая точка $p_{0}$ является простой двойной точкой. Мы можем считать, что гиперплоскость $x_{0}=0$ не касается многообразия $X_{0}$. Проективное пространство с однородньми координатами $\left(x_{1}: x_{2}: x_{3}: x_{4}\right)$ будем обозначать через $\mathbb{P}^{3}$.

Пусть $C$ - это кривая в $\mathbb{P}^{3}$, которая является пересечением квадрики $Q \subset \mathbb{P}^{3}$, заданной уравнением

$$
\sum_{1 \leqslant i, j \leqslant 4} a_{i j} x_{i} x_{j}=0
$$

и кубики $S$, заданной уравнением

$$
\sum_{1 \leqslant i, j, k \leqslant 4} b_{i j k} x_{i} x_{j} x_{k}=0
$$


Она является неособой кривой рода 4 . Пусть $F_{0}$ - поверхность $Ф$ ано кубики $X_{0}$, которая параметризует множество прямых на этой кубике, а $C^{(2)}$ - симметрический квадрат кривой $C$. Тогда в [1] доказывается существование короткой точной последовательности

$$
0 \rightarrow H^{q-1}(C, \mathbb{Z}) \rightarrow H^{q}\left(F_{0}, \mathbb{Z}\right) \rightarrow H^{q}\left(C^{(2)}, \mathbb{Z}\right) \rightarrow 0
$$

при каждом $q$.

ЛЕмма 1.1. При кажсом $q$ группа когомологий $H^{q}\left(F_{0}, \mathbb{Z}\right)$ свободная.

ДокАЗАТЕЛЬСТво. Из точных последовательностей (4) вытекает, что достаточно проверить отсутствие кручения в группах $H^{q}\left(C^{(2)}, \mathbb{Z}\right)$. Так как поверхность $C^{(2)}$ неособая, достаточно установить отсутствие кручения в группе $H^{2}\left(C^{(2)}, \mathbb{Z}\right)$. Для этого мы будем применять эквивариантные когомологии Гротендика. Пусть $g: C^{2} \rightarrow C^{2}-$ инволющия на декартовом квадрате, которая переставляет сомножители, а $G=\{1, g\}-$ группа второго порядка, порожденная этой инволюцией. Тогда рассмотрим эквивариантные когомологии Гротендика $H^{q}\left(C^{2}, G ; \mathbb{Z}\right)$. Имеются две спектральные последовательности, сходящиеся к этим когомологиям (см. подробности в [2]):

$$
\begin{gathered}
I_{2}^{p, q}=H^{p}\left(C^{2} / G, \mathscr{H}^{q}(G, \mathbb{Z})\right) \Longrightarrow H^{p+q}\left(C^{2}, G ; \mathbb{Z}\right), \\
I I_{2}^{p, q}=H^{p}\left(G, H^{q}\left(C^{2}, \mathbb{Z}\right)\right) \Longrightarrow H^{p+q}\left(C^{2}, G ; \mathbb{Z}\right) .
\end{gathered}
$$

Так как (см., например, [2], [3])

$$
\mathscr{H}^{q}(G, \mathbb{Z})= \begin{cases}\mathbb{Z}, & q=0, \\ \left.(\mathbb{Z} / 2)\right|_{\left(C^{2}\right)^{G}}, & q=2 k, \\ 0, & q=2 k+1,\end{cases}
$$

и $C^{2} / G=C^{(2)},\left(C^{2}\right)^{G}=\Delta=C$ - диагональ, из первой спектральной последовательности получается точная последовательность (см., например, [7])

$$
0 \rightarrow H^{2}\left(C^{(2)}, \mathbb{Z}\right) \rightarrow H^{2}\left(C^{2}, G ; \mathbb{Z}\right) \rightarrow H^{0}(\Delta, \mathbb{Z} / 2)
$$

Заметим, что группа $I I_{2}^{2,0}=H^{2}\left(G, H^{0}\left(C^{2}, \mathbb{Z}\right)\right)=\mathbb{Z} / 2$ содержится в $H^{2}\left(C^{2}, G ; \mathbb{Z}\right)$ и отображается изоморфно на группу $H^{0}(\Delta, \mathbb{Z} / 2)=\mathbb{Z} / 2$ (см., например, [7]). Поэтому получаем вложение

$$
H^{2}\left(C^{(2)}, \mathbb{Z}\right) \hookrightarrow H^{2}\left(C^{2}, G ; \mathbb{Z}\right) / I I_{2}^{2,0}
$$

Заметим теперь, что

$$
I I_{2}^{1,1}=H^{1}\left(G, H^{1}\left(C^{2}, \mathbb{Z}\right)\right)=0 .
$$

Действительно, групша $H^{1}\left(C^{2}, \mathbb{Z}\right)$ состоит из линейных комбинаций $n(a \otimes 1)+m(1 \otimes b)$, где $a, b \in H^{1}(C, \mathbb{Z}), n, m \in \mathbb{Z}$, а инволюция

$$
g^{*}: H^{1}\left(C^{2}, \mathbb{Z}\right) \rightarrow H^{1}\left(C^{2}, \mathbb{Z}\right)
$$

действует по правилу

$$
g^{*}(n(a \otimes 1)+m(1 \otimes b))=n(1 \otimes a)+m(b \otimes 1) .
$$


Осталось увидеть, что из этой формулы следует равенство

$$
\left\{a \in H^{1}\left(C^{2}, \mathbb{Z}\right) \mid g^{*}(a)=-a\right\}=\left\{b-g^{*}(b) \mid b \in H^{1}\left(C^{2}, \mathbb{Z}\right)\right\}
$$

поэтому

$$
H^{1}\left(G, H^{1}\left(C^{2}, \mathbb{Z}\right)\right)=\frac{\left\{a \in H^{1}\left(C^{2}, \mathbb{Z}\right) \mid g^{*}(a)=-a\right\}}{\left\{b-g^{*}(b) \mid b \in H^{1}\left(C^{2}, \mathbb{Z}\right)\right\}}=0 .
$$

Следовательно, группа $H^{2}\left(C^{2}, G ; \mathbb{Z}\right) / I I_{2}^{2,0}$ содержится в групе $I I_{2}^{0,2}=\left(H^{2}\left(C^{2}, \mathbb{Z}\right)\right)^{G}$, поэтому является свободной. Лемма доказана.

Для поверхности $\Phi$ ано $F$ неособой кубики $X$ в [1] доказано существование короткой точной последовательности

$$
0 \rightarrow H^{q}\left(F_{0}, \mathbb{Z}\right) \rightarrow H^{q}(F, \mathbb{Z}) \rightarrow H^{q-1}(C, \mathbb{Z}) \rightarrow 0
$$

при каждом $q$. Из этой точной последовательности и леммы 1.1 вытекает

ЛЕмма 1.2. При кажсом $q$ группа когомологий $H^{q}(F, \mathbb{Z})$ свободная.

Из точных последовательностей (4), (5) вытекают равенства

$$
H^{1}(F, \mathbb{Z})=\mathbb{Z}^{10}, \quad H^{2}(F, \mathbb{Z})=\mathbb{Z}^{45}
$$

В работе [1] выбирается специальный базис $\chi, \delta, \eta_{1}, \ldots, \eta_{8}$ групшы $H^{1}(F, \mathbb{Z})$, для которого доказьваются следующие соотношения:

$$
\begin{aligned}
& \left(\chi \cup \delta \cup \eta_{k} \cup \eta_{k+4},[F]\right)=1 \quad \text { при } 1 \leqslant k \leqslant 4 \text {; } \\
& \left(\chi \cup \delta \cup \eta_{k} \cup \eta_{l},[F]\right)=0 \quad \text { при } l>k, l \neq k+4 ; \\
& \left(\chi \cup \eta_{k} \cup \eta_{l} \cup \eta_{m},[F]\right)=0 \quad \text { для всех } k, l, m \text {; } \\
& \left(\delta \cup \eta_{k} \cup \eta_{l} \cup \eta_{m},[F]\right)=0 \quad \text { для всех } k, l, m \text {; } \\
& \left(\eta_{k} \cup \eta_{k+4} \cup \eta_{l} \cup \eta_{l+4},[F]\right)=1 \quad \text { при } 1 \leqslant k<l \leqslant 4 \text {; } \\
& \left(\eta_{p} \cup \eta_{q} \cup \eta_{r} \cup \eta_{s},[F]\right)=0 \quad \text { для всех других } p, q, r, s \text {. }
\end{aligned}
$$

Кроме того доказывается, что класс когомологий

$$
\omega=\frac{1}{2}\left(\chi \cup \delta+\sum_{1}^{4} \eta_{k} \cup \eta_{k+4}\right)
$$

принадлежит группе $H^{2}(F, \mathbb{Z})$, т.е. является целым, причем этот класс имеет следующий геометрический смысл. Пусть $l$-произвольная общая прямая на $X$; тогда множество прямых на $X$, пересекающих $l,-$ неособая кривая $D_{l}$ на $F$. Класс когомологий, двойственный циклу $\left[D_{l}\right]$, оказывается равен классу $\omega$. 
ЛЕмма 1.3. Классы когомологий

$$
\begin{gathered}
\omega_{1}=\omega, \quad \omega_{2}=\eta_{1} \cup \eta_{5}, \quad \omega_{3}=\eta_{2} \cup \eta_{6}, \quad \omega_{4}=\eta_{3} \cup \eta_{7}, \quad \omega_{5}=\eta_{4} \cup \eta_{8}, \\
\omega_{6}=\chi \cup \eta_{1}, \quad \omega_{7}=\chi \cup \eta_{2}, \quad \ldots, \quad \omega_{13}=\chi \cup \eta_{8}, \quad \omega_{14}=\delta \cup \eta_{1}, \quad \omega_{15}=\delta \cup \eta_{2}, \quad \ldots, \\
\omega_{21}=\delta \cup \eta_{8}, \quad \omega_{22}=\eta_{1} \cup \eta_{2}, \quad \omega_{23}=\eta_{1} \cup \eta_{3}, \quad \omega_{24}=\eta_{1} \cup \eta_{4}, \\
\omega_{25}=\eta_{1} \cup \eta_{6}, \quad \omega_{26}=\eta_{1} \cup \eta_{7}, \quad \omega_{27}=\eta_{1} \cup \eta_{8}, \quad \omega_{28}=\eta_{2} \cup \eta_{3}, \\
\omega_{29}=\eta_{2} \cup \eta_{4}, \quad \omega_{30}=\eta_{2} \cup \eta_{5}, \quad \omega_{31}=\eta_{2} \cup \eta_{7}, \quad \omega_{32}=\eta_{2} \cup \eta_{8}, \quad \ldots, \\
\omega_{43}=\eta_{6} \cup \eta_{7}, \quad \omega_{44}=\eta_{6} \cup \eta_{8}, \quad \omega_{45}=\eta_{7} \cup \eta_{8}
\end{gathered}
$$

образуют базис свободной группь $H^{2}(F, \mathbb{Z})$.

ДокАЗАТЕЛЬСтво. Рассмотрим билинейную симметрическую форму на свободной группе $H^{2}(F, \mathbb{Z})$

$$
(\alpha, \beta)=(\alpha \cup \beta,[F]) .
$$

Из второго равенства в (6) следует, что достаточно проверить, что определитель матрицы $\left\|\left(\omega_{i}, \omega_{i}\right)\right\|$ равен \pm 1 . Эта матрища разбивается на блоки

$$
\left\|\begin{array}{cc}
A & C \\
C^{t} & B
\end{array}\right\|,
$$

где

$$
A=\left\|\begin{array}{lllll}
5 & 2 & 2 & 2 & 2 \\
2 & 0 & 1 & 1 & 1 \\
2 & 1 & 0 & 1 & 1 \\
2 & 1 & 1 & 0 & 1 \\
2 & 1 & 1 & 1 & 0
\end{array}\right\|,
$$

$C$ - нулевая матрица размера $5 \times 40$, а $B$ - матрица размера $40 \times 40$, имеющая в каждой строке и в каждом столбце по одному ненулевому элементу, причем вида \pm 1 . Определитель матрищы $B$ равен, с точностью до знака, произведению еененулевых элементов \pm 1 , поэтому он сам равен \pm 1 . Непосредственная проверка показывает, что определитель матрицы $A$ равен 1. Лемма доказана.

\section{ЛЕмма 1.4. Классы когомологий}

$$
\begin{gathered}
\alpha_{1}=\chi \cup \delta \cup \eta_{1}, \quad \alpha_{2}=\chi \cup \delta \cup \eta_{5}, \quad \alpha_{3}=\chi \cup \eta_{1} \cup \eta_{5}, \quad \alpha_{4}=\delta \cup \eta_{1} \cup \eta_{5}, \\
\alpha_{5}=\eta_{2} \cup \eta_{1} \cup \eta_{5}, \quad \alpha_{6}=\eta_{3} \cup \eta_{1} \cup \eta_{5}, \quad \alpha_{7}=\eta_{4} \cup \eta_{1} \cup \eta_{5}, \\
\alpha_{8}=\eta_{6} \cup \eta_{1} \cup \eta_{5}, \quad \alpha_{9}=\eta_{7} \cup \eta_{1} \cup \eta_{5}, \quad \alpha_{10}=\eta_{8} \cup \eta_{1} \cup \eta_{5}
\end{gathered}
$$

образуют базис свободной группь $H^{3}(F, \mathbb{Z})=\mathbb{Z}^{10}$.

ДокАЗАТЕЛЬСтво. Положим $\beta_{1}=\chi, \beta_{2}=\delta, \beta_{3}=\eta_{1}, \ldots, \beta_{10}=\eta_{8}$. Это базис группы $H^{1}(F, \mathbb{Z})=\mathbb{Z}^{10}$. Рассмотрим теперь матрицу $M=\left\|\left(\alpha_{i} \cup \beta_{j},[F]\right)\right\|$ размера $10 \times 10$. Непосредственно проверяется с помощью соотношений выше, что эта матрица имеет в каждой строке и в каждом столбце по одному ненулевому элементу, причем вида \pm 1 . Ее определитель равен, с точностью до знака, произведению ее ненулевых элементов \pm 1 , поэтому он сам равен \pm 1 . Так как элементы $\beta_{1}, \ldots, \beta_{10}$ образуют базис 
группы $H^{1}(F, \mathbb{Z})$, из этого факта про матрицу $M$ следует, что элементы $\alpha_{1} \ldots, \alpha_{10}$ тоже образуют базис групшы $H^{3}(F, \mathbb{Z})$. Лемма доказана.

В этом пункте нам остается поговорить об отображении Абеля-Якоби. Пусть $P$ подмногообразие произведения $F \times X$, состоящее из пар $(l, x)$ таких, что прямая $l$ содержит точку $x$. Тогда две проекции прямого произведения определяют диаграмму

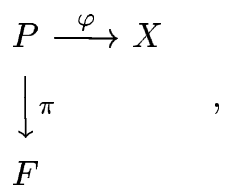

где $\pi: P \rightarrow F-$ локально тривиальное расслоение со слоем прямая, а $\varphi: P \rightarrow X-$ конечнолистное накрытие. Диаграмма (7) назьвается семейством Фано кубики. Она позволяет определить гомоморфизм групп гомологий

$$
\Phi: H_{1}(F, \mathbb{Z}) \rightarrow H_{3}(X, \mathbb{Z})
$$

который называется отображением иилиндра и по определению равен композиции отображений

$$
H_{1}(F, \mathbb{Z}) \stackrel{\pi^{-1}}{\longrightarrow} H_{3}(P, \mathbb{Z}) \stackrel{\varphi_{*}}{\longrightarrow} H_{3}(X, \mathbb{Z}),
$$

где отображение $\pi^{-1}$ сопоставляет замкнутой кривой $\gamma$ на $F$ ее прообраз $\pi^{-1}(\gamma)$, которьй является цилиндром. Справедлива

ЛЕмма 1.5. Гомоморфизм иилиндра

$$
\Phi: H_{1}(F, \mathbb{Z}) \rightarrow H_{3}(X, \mathbb{Z})
$$

является изоморфизмом.

ДокАЗАТЕЛЬСТво. Отображение цилиндра индуцирует гомоморфизм Абеля-Якоби

$$
\Phi: A(F) \rightarrow J(X)
$$

многообразия Альбанезе поверхности $S$ в промежуточный якобиан тела $X$. В работе [1] показано, что гомоморфизм Абеля-Якоби является изоморфизмом. Следовательно, он задает изоморфизм фундаментальных групा соответствующих комплексных торов

$$
\pi_{1}(A(F)) \rightarrow \pi_{1}(J(X))
$$

Так как групшы $H_{1}(F, \mathbb{Z}), H_{3}(X, \mathbb{Z})$ свободные, они совпадают с этими фундаментальными группами. Лемма доказана. 
2. Доказательство основного результата. Прежде всего заметим, что наша кубика $(X, \tau)$ будет $M$-многообразием, если и только если гомоморфизм

$$
\tau^{*}: H^{3}\left(V, \mathbb{F}_{2}\right) \rightarrow H^{3}\left(V, \mathbb{F}_{2}\right)
$$

будет тождественным отображением. Это вытекает из нечетности степени кубики (см., например, [3], [8]). Так как множество $\mathbb{R} F$ непустое, то, чтобы поверхность $\Phi$ ано $(F, \tau)$ была $M$-поверхностью, необходимо и достаточно вьполнение следующих условий:

1) гомоморфизм $\tau^{*}: H^{q}\left(F, \mathbb{F}_{2}\right) \rightarrow H^{q}\left(F, \mathbb{F}_{2}\right)$ является тождественным отображением при $q=1,2$;

2) дифференциалы $d_{2}^{0,2}, d_{2}^{0,3}, d_{3}^{0,3}$ в спектральной последовательности

$$
I I_{2}^{p, q}=H^{p}\left(G, H^{q}\left(F, \mathbb{F}_{2}\right)\right) \Longrightarrow H^{p+q}\left(F, G ; \mathbb{F}_{2}\right)
$$

равны нулю (тогда все дифференциалы будут нулевые, см., например, [3], [8]).

Построение отображения цилиндра

$$
\Phi: H_{1}\left(F, \mathbb{F}_{2}\right) \rightarrow H_{3}\left(X, \mathbb{F}_{2}\right)
$$

показывает, что оно коммутирует с комплексньм сопряжением. Поэтому из леммы 1.5 следует, что гомоморфизм

$$
\tau^{*}: H^{3}\left(X, \mathbb{F}_{2}\right) \rightarrow H^{3}\left(X, \mathbb{F}_{2}\right)
$$

будет тождественным отображением тогда и только тогда, когда гомоморфизм

$$
\tau^{*}: H^{1}\left(F, \mathbb{F}_{2}\right) \rightarrow H^{1}\left(F, \mathbb{F}_{2}\right)
$$

тождественный. Следовательно, если поверхность $\Phi$ ано $F$ является $M$-поверхностью, то кубика $X$ будет $M$-многообразием. Переходим к доказательству обратного утверждения. Для этого нужно проверить выполнение условий 1), 2).

Если кубика является $M$-многообразием, то на групе $H^{3}\left(X, \mathbb{F}_{2}\right)$ инволюция $\tau^{*}$ действует тривиально, поэтому в силу леммы 1.5 она действует тривиально и на группе $H^{1}\left(F, \mathbb{F}_{2}\right)$. Применим лемму 1.3 ; тогда получим, что инволюция $\tau^{*}$ действует тривиально на базисных элементах $\omega_{2}(\bmod 2), \ldots, \omega_{45}(\bmod 2)$ группы $H^{2}\left(F, \mathbb{F}_{2}\right)$. Заметим, что инволюция действует тривиально и на элементе $\omega_{1}(\bmod 2)=\left[D_{l}\right]^{*}(\bmod 2)$. Действительно, если взять вещественную прямую $l$ на кубике, то дивизор $D_{l}$ инвариантен относительно инволюции $\tau$. Таким образом, первое условие проверено; переходим к проверке второго.

Прежде всего заметим, что дифференциалы $d_{2}^{p, 1}$ равны нулю, так как множество $\mathbb{R} F$ непустое (см., например, [3]), а дифференциалы $d_{3}^{p, 1}$ всегда равны нулю по соображениям размерности. Далее введем следующее обозначение. Если $a \in H^{q}(F, \mathbb{Z})$, то элемент $a(\bmod 2) \in H^{q}\left(F, \mathbb{F}_{2}\right)$ будем обозначать через $a^{\prime}$. Так как инволюция $\tau^{*}$ действует на $H^{q}\left(F, \mathbb{F}_{2}\right)$ тривиально, то выполняется равенство $I I_{2}^{p, q}=H^{q}\left(F, \mathbb{F}_{2}\right)$. Прежде всего нужно показать, что $d_{2}\left(\omega_{i}^{\prime}\right)=0$ при $i=1, \ldots, 45$. Элемент $\omega_{1}^{\prime}$ получается из дивизора, инвариантного относительно инволюции $\tau$, поэтому $d_{2}\left(\omega_{1}^{\prime}\right)=0$ (см., например, [4]-[6]). Каждый элемент $\omega_{i}^{\prime}$ при $i=2, \ldots, 45$ равен произведению элементов из $H^{1}\left(F, \mathbb{F}_{2}\right)$, поэтому $d_{2}\left(\omega_{i}^{\prime}\right)=0$ согласно правилу Лейбница. Например, имеем равенства

$$
d_{2}\left(\omega_{2}^{\prime}\right)=d_{2}\left(\eta_{1}^{\prime} \cup \eta_{5}^{\prime}\right)=d_{2}\left(\eta_{1}^{\prime}\right) \cup \eta_{5}^{\prime}+\eta_{1}^{\prime} \cup d_{2}\left(\eta_{5}^{\prime}\right)=0,
$$


так как $d_{2}\left(\eta_{1}^{\prime}\right)=0, d_{2}\left(\eta_{5}^{\prime}\right)=0$. Из лемм $1.2,1.3$ следует, что элементы $\omega_{1}^{\prime}, \ldots, \omega_{45}^{\prime}$ образуют базис пространства $H^{2}\left(F, \mathbb{F}_{2}\right)$, поэтому дифференциал

$$
d_{2}^{0,2}: I I_{2}^{0,2} \rightarrow I I_{2}^{2,1}
$$

равен нулю. Равенство нулю дифференциалов

$$
d_{2}^{0,3}: I I_{2}^{0,3} \rightarrow I I_{2}^{2,2}, \quad d_{3}^{0,3}: I I_{3}^{0,3} \rightarrow I I_{3}^{3,1}
$$

доказьвается аналогично, а именно, с помощю леммы 1.4 и правила Лейбница. Таким образом, проверено второе условие максимальности поверхности Фано. Следовательно, теорема 0.1 полностью доказана.

\section{СПИСОК ЦИТИРОВАННОЙ ЛИТЕРАТУРЫ}

[1] Clemens C. H., Griffits P. A. The intermediate Jacobian of the cubic threefold // Ann. Math. 1972. V. 95. P. 281-356.

[2] Гротендик А. О некоторых вопросах гомологической алгебры. М.: ИЛ, 1961.

[3] Краснов В.А. Неравенства Гарнака-Тома для отображений вещественных алгебраических многообразий // Изв. АН СССР. Сер. матем. 1983. Т. 47. № 2. C. $268-297$.

[4] Краснов В.А. Характеристические классы векторных расслоений на вещественном алгебраическом многообразии // Изв. АН СССР. Сер. матем. 1991. Т. 55. № 4. С. 716-746.

[5] К раснов В.А. Алгебраические циклына вешественном алгебраическом $G M$-многообразии и их приложения // Изв. РАН. Сер. матем. 1993. Т. 57. № 4. С. 153-173.

[6] Краснов В.А. Об эквивариантных когомологиях Гротендика вещественного алгебраического многообразия и их приложениях // Изв. РАН. Сер. матем. 1994. Т. 58. № 3. С. 36-52.

[7] Краснов В.А. Эквивариантные когомологии вещественной алгебраической поверхности и их приложения // Изв. РАН. Сер. матем. 1996. Т. 60. №6. С. 101-126.

[8] Краснов В. А. Вещественные алгебраические $G M$-многообразия // Изв. РАН. Сер. матем. 1998. T. 62. № 3. C. 39-66. 